\title{
Development of a novel and low-cost anthropomorphic pelvis phantom for 3D dosimetry in radiotherapy
}

\author{
Somayyeh Babaloui, PhD 1.2, Shakardokht Jafari, PhD2,3, Wojciech Polak, PhD³, Mahdi Ghorbani, PhD ${ }^{4}$, \\ Michael WJ Hubbard, PhD², Annika Lohstroh, PhD², . Alireza Shirazi, PhD', Ramin Jaberi, PhD2,6 \\ 'Department of Medical Physics and Biomedical Engineering, Faculty of Medicine, Tehran University of Medical Sciences, Tehran, Iran, \\ ${ }^{2}$ Department of Physics, University of Surrey, Guildford, United Kingdom, ${ }^{3}$ Medical Physics Department, Portsmouth Hospitals NHS Trust, \\ Portsmouth, United Kingdom, ${ }^{B}$ Biomedical Engineering and Medical Physics Department, Faculty of Medicine, Shahid Beheshti University

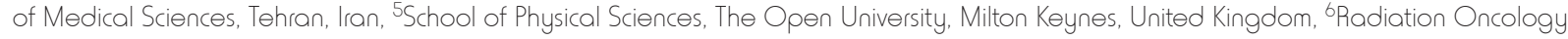 \\ Research Centre (RORC), Cancer Institute, Tehran University of Medical Sciences, Tehran, Iran
}

\begin{abstract}
Purpose: The aim of this study was to construct a low-cost, anthropomorphic, and 3D-printed pelvis phantom and evaluate the feasibility of its use to perform 3D dosimetry with commercially available bead thermoluminescent dosimeters (TLDs).

Material and methods: A novel anthropomorphic female phantom was developed with all relevant pelvic organs to position the bead TLDs. Organs were 3D-printed using acrylonitrile butadiene styrene. Phantom components were confirmed to have mass density and computed tomography (CT) numbers similar to relevant tissues. To find out clinically required spatial resolution of beads to cause no perturbation effect, TLDs were positioned with 2.5, 5, and 7.5 mm spacing on the surface of syringe. After taking a CT scan and creating a 4-field conformal radiotherapy plan, 3 dose planes were extracted from the treatment planning system (TPS) at different depths. By using a 2D-gamma analysis, the TPS reports were compared with and without the presence of beads. Moreover, the bead TLDs were placed on the organs' surfaces of the pelvis phantom and exposed to high-dose-rate (HDR) ${ }^{60}$ Co source. TLDs' readouts were compared with the TPS calculated doses, and dose surface histograms (DSHs) of organs were plotted.

Results: 3D-printed phantom organs agreed well with body tissues regarding both their design and radiation properties. Furthermore, the 2D-gamma analysis on the syringe showed more than $99 \%$ points passed 3\%- and 3-mm criteria at different depths. By calculating the integral dose of DSHs, the percentage differences were $-1.5 \%, 2 \%, 5 \%$, and $10 \%$ for uterus, rectum, bladder, and sigmoid, respectively. Also, combined standard uncertainty was estimated as $3.5 \%(k=1)$.

Conclusions: A customized pelvis phantom was successfully built and assessed to confirm properties similar to body tissues. Additionally, no significant perturbation effect with different bead resolutions was presented by the external TPS, with $0.1 \mathrm{~mm}$ dose grid resolution.

Key words: anthropomorphic phantom, 3D dosimetry, silica bead TLDs, radiotherapy.

\section{Purpose}

Medical phantoms are artificial representations of human body structures, which are used in training, auditing, and quality assurance in medicine $[1,2]$. The aim of using them in radiotherapy (RT) is to ensure precision dose delivery to the patient with geometric accuracy [2] Depending on their application, phantoms are made of different compositions and geometries: from simple cylinders of liquid or solid homogenous materials, such as slab phantoms, to complicated multi-material prototypes [3]. The correspondence between dosimetric phantoms and tissue behavior is very important in RT, because any radiological inconsistency between the phantom and patient's body, may affect the treatment results and even lead to poor treatment outcomes [2,4]. Phantoms, which
Address for correspondence: Ramin Jaberi, PhD, Radiation Oncology Research Centre (RORC), Cancer Institute, Tehran University of Medical Sciences, Tehran, Iran, phone: +9891 23599974, fax: +98 21 8609060, e-mail: rjaberi@tums.ac.ir, rjaberi1345@gmail.com; Alireza Shirazi, Department of Medical Physics and Biomedical Engineering, Faculty of Medicine, Tehran University of Medical Sciences, Tehran, Iran, phone: +98 91 21484769, fax: +98 21 64053242, e-mail: shirazia@sina.tums.ac.ir
Received: 17.04.2020

Accepted: 29.07.2020

Published: 30.10.2020 
better indicate tissue properties are called anthropomorphic phantoms, and their development in different applications are an ongoing challenge. Anthropomorphic phantoms are representative of human body in terms of size, shape, and functions; they are built of structures with tissue equivalent material, which represent radiological properties of internal organs [2]. In past decades, the usage of them was prevalent and beneficial for dosimetric inter-comparisons as well as verification procedures and patient-specific quality control (PSQC) [5,6]. Moreover, the utilization of such phantoms in advanced RT (e.g., intensity-modulated radiation therapy (IMRT) and volumetric modulated arc therapy (VMAT)), due to the lack of lateral electron equilibrium and steep dose gradient in small fields, is fundamental [7]. In recent years, several studies have shown the advantages of 3D-printed phantoms for the use in RT and PSQC $[8,9,10]$.

The aim of this study was to develop a novel and lowcost anthropomorphic pelvis phantom with 3D-printed organs and to evaluate the feasibility of its usage to perform 3D dosimetry in RT. Micro-silica bead thermoluminescent dosimeters (TLDs) are placed on the surface of internal organs and inside the body cavity for 3D dose mapping measurements. Although, there are some commercially available or home-made gynecologic (GYN) phantoms $[11,12,13,14]$, there is no affordable dosimetric GYN phantom, with tissue equivalent composition and removable organs, especially for brachytherapy (BT). Furthermore, almost all of the existing phantoms, such as Rando phantoms, are used to only determine point doses in organs or to perform 2D dosimetry. 3D dosimetry, with the required positioning accuracy is still not possible. In this work, a female pelvis phantom was produced, with a simple method using available tissue equivalent materials to obtain 3D dose distribution.

\section{Material and methods}

\section{Micro-silica bead thermoluminescent dosimeters and reading system}

A batch of 500 micro-silica beads (TrueInvivo Ltd., UK), $1.5 \pm 0.1 \mathrm{~mm}$ in diameter, $1.0 \pm 0.1 \mathrm{~mm}$ in thickness, and $3.7 \pm 0.1 \mathrm{mg}$ in weight, with material composition (by weight), including C $-8.93 \%, \mathrm{O}-42.18 \%$, Na $-10.55 \%$, $\mathrm{Al}-1.3 \%$, $\mathrm{Si}-33.62 \%, \mathrm{~K}-1.09 \%$, $\mathrm{Ca}-1.92 \%$, and $\mathrm{Fe}-$ $0.37 \%$, was employed for this study. These dosimeters were selected for the dosimetry because of several of characteristics, such as small size, with a chemically inert nature, inexpensive, easy to use and reusable, with a fading rate of $10 \%$ at 30 days after irradiation and high thermoluminescence (TL) light transparency, high sensitivity, and a large dynamic dose range that remains linear $\left(R^{2} \geq 0.999\right)$ from 1 cGy to 25 Gy $[15,16,17,18]$.

A Toledo 654 TLD reader (D.A. Pitman Instruments Ltd., UK) was employed to readout the bead TLDs manually at the University of Surrey. In order to obtain the optimum full glow-curve by using the present system, the readout cycle was initiated, when the planchet reached a temperature of $160^{\circ} \mathrm{C}$, followed by heating to a maximum of $350^{\circ} \mathrm{C}$ at a ramp rate of $20^{\circ} \mathrm{C} / \mathrm{s}$.
The individual calibration factors of silica beads were determined with a $6 \mathrm{MV}$ photon beam $(\mathrm{TPR} 20 / 10=0.662)$, using a Varian Clinac $^{\circledR} 2100 \mathrm{C}$ linear accelerator (Varian ${ }^{\circledR}$ Medical Systems, Palo Alto, CA, USA) [16].

This relative coefficient accounts for the variation of individual detector sensitivities in the same TLD batch with respect to the physical properties, such as mass and dopant concentration, and is defined by the following equation:

$$
\mathrm{ECC}_{j}=\frac{\langle\mathrm{TLR}\rangle}{\mathrm{TLR}_{j}}
$$

where ECC $j$ is the ECC of individual bead dosimeter $j$, TLRj is the TL response of dosimeter $j$, and 〈TLR > is the mean TLR of irradiated dosimeters in the same irradiation field.

This factor is energy-independent in the selected range [18] and therefore, the bead TLDs were calibrated with a $6 \mathrm{MV}$ photon beam. The final reading of the silica beads were obtained by multiplying the raw output values of TLDs by their individual calibration coefficient.

\section{Beam perturbation effect}

According to Jafari et al. [18], the effective atomic number of silica bead TLDs is equal to 10.6, which is close to the effective atomic number of bone $\left(Z_{\text {eff }}=12.31\right)$ [19]. To estimate the beam perturbation effect of bead TLDs and to find the best clinically relevant spatial resolution (SR), which was required to arrange the beads on the phantom organs' surfaces, an experiment was performed and the effect of the presence of silica beads on beam attenuation was evaluated. The spatial resolution of silica beads refers to the separation of them to each other.

A 5-cc syringe was chosen, and some holes were made across its whole surface. The holes had $2.5 \mathrm{~mm}$ center point spacing (Figure 1A). The reason for selecting the syringe was because its material is suitable to observe the silica beads individually on computed tomography (CT) images. The silica beads were placed on the surface of the syringe, with three different spacing: $2.5 \mathrm{~mm}$ (high), $5 \mathrm{~mm}$ (medium), and $7.5 \mathrm{~mm}$ (low) resolution. The syringe was positioned on two legs in a reproducible position in a water tank (Figure 1B).

The water tank was filled with water and positioned in the RT simulator CT scanner (Figure 1C), and a helical scan of $2 \mathrm{~mm}$ slice thickness was taken. Also, one series of images was obtained of the syringe without the beads. DICOM images were imported into the Pinnacle planning system (version 16.02.60004, Philips Healthcare, Andover, USA). From the treatment planning system (TPS), 3 dose planes (in 2D format) were extracted at different depths, in the middle (source to plane distance $(\mathrm{SPD})=99 \mathrm{~cm})$, bottom $(\mathrm{SPD}=100 \mathrm{~cm})$, and $2 \mathrm{~cm}$ below $(\mathrm{SPD}=102 \mathrm{~cm})$ the syringe for each TLD's arrangement. By using a 2D-gamma analysis, the TPS reports with and without beads were compared.

Similarly, some artificial "organs", including rectum, bladder, and uterus were contoured on the images obtained with different beads resolution and without them; rectum was represented by the syringe, the rectal wall by the layer of silica beads over the syringe, the bladder, 

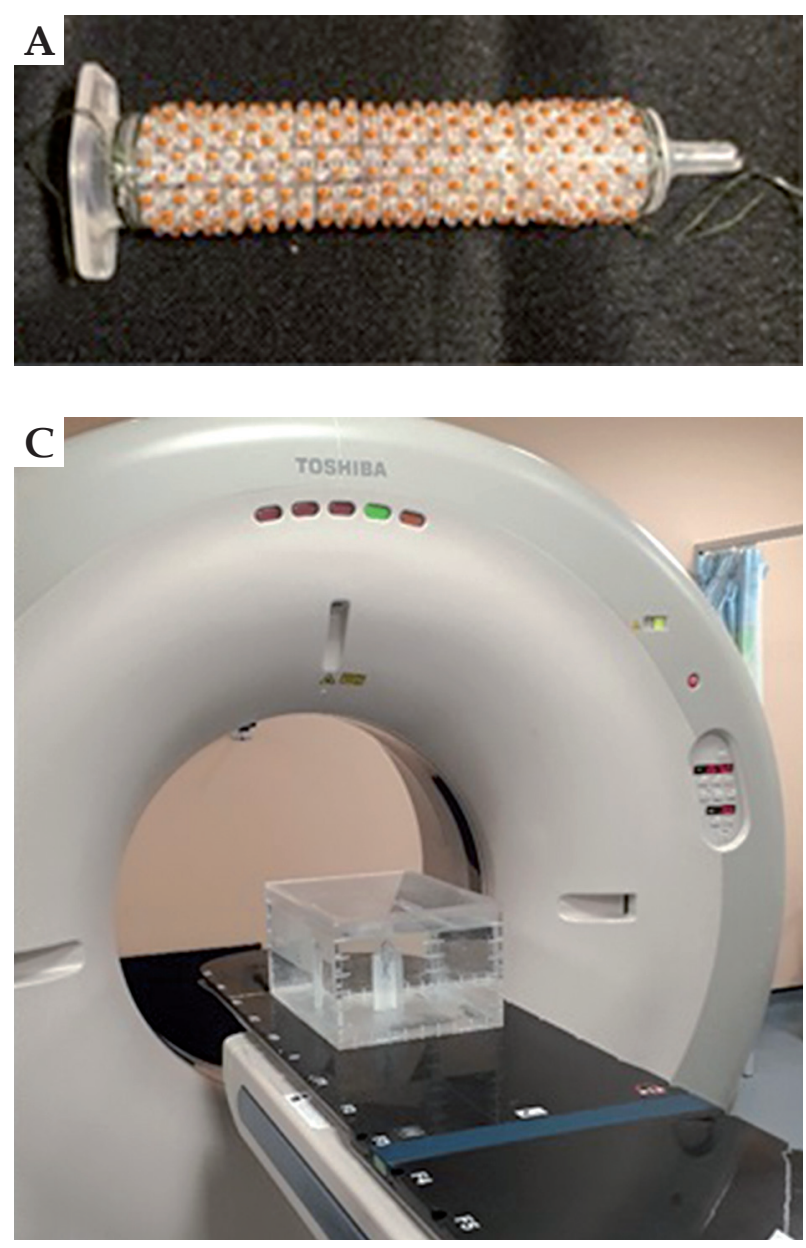

uterus (clinical target volume - CTV), and uterus $+5 \mathrm{~mm}$ margin (planning target volume - PTV). A 4-field (box) conformal treatment plan was performed, with a dose of $45 \mathrm{~Gy} / 20 \mathrm{fr}$. The treatment plan was the same for different silica bead SR.

\section{Phantom design criteria}

Dosimetric phantoms in radiotherapy have some common requirements, such as suitability in terms of transport, radiological tissue equivalence, considering physical and electron densities, anatomical accuracy in terms of organs' geometry, and positioning. In addition, the other key criteria that are addressed by the current phantom included light weight and portability, manufacturing from non-toxic and non-degradable materials, visually distinguishable organs for the TPS, volume delineation and dose calculation, ability to accommodate a large number of silica beads (about 920 silica beads) on the organs' surfaces, reproducible rapid assembly and disassembly, and finally, budget and timeline constraints satisfaction.

\section{Phantom features}

The presented anthropomorphic phantom has been developed for experimental studies of cervix and uterus

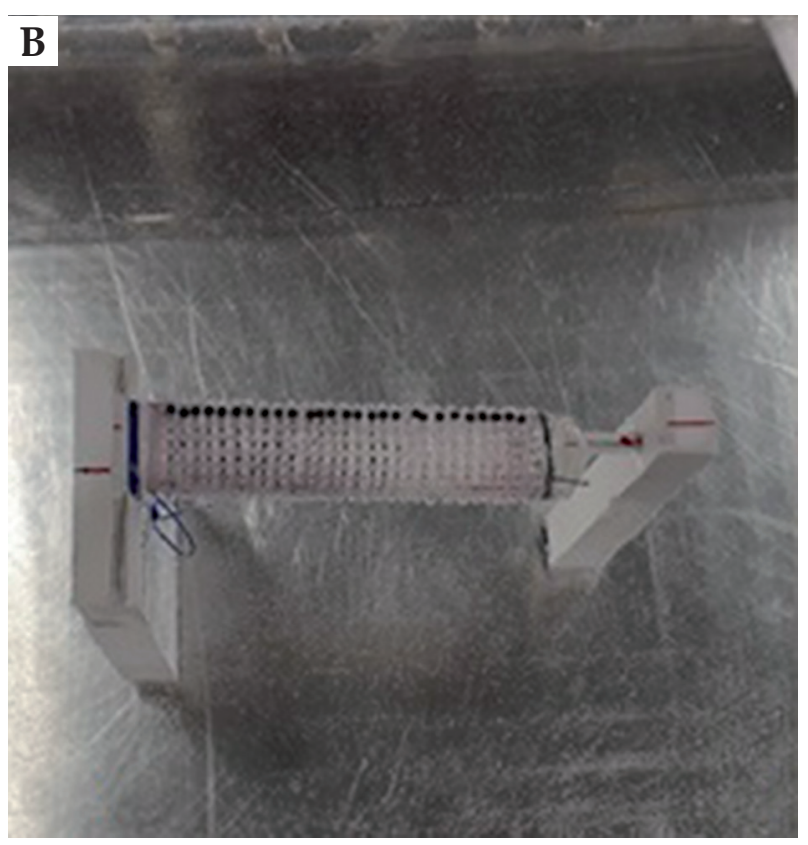

Fig. 1. A) Syringe with a $2.5 \mathrm{~mm}$ spacing arrangement of silica bead TLDs, B) The syringe located on the two legs into the water tank, C) The positioning of the water tank for CT scanning

treatments, and corresponds to a female pelvis of average body size. It consists of a pelvic skeleton with interskeleton cartilages, uterus, bladder, rectum, and sigmoid. For BT usage, a tandem-ring applicator is located in place and for external RT, it is removed. The anthropomorphic shape of organs represents a realistic clinical situation that is important during patient's simulation. All components of the phantom are metal-free to ensure CT and magnetic resonance imaging (MRI) compatibility.

The phantom construction procedure consisted of: 1. Water tank and base plate preparation, 2. The pelvic bone preparation, and 3 . Internal organs preparation. The following paragraphs describe in detail the phantom construction process.

\section{Water tank and base plate preparation}

For placing the phantom in the water, a water tank with dimensions of 45,33, and $24 \mathrm{~cm}^{3}$ (length, width, and height, respectively) was made. The water tank dimensions were sufficiently large to provide clinically relevant scatter conditions.

Also, a rectangular base plate with dimension of 29.5, 19.5, and $18 \mathrm{~cm}^{3}$ (length, width, and height, respectively) was made of Perspex material and nylon screws (RS Components, UK) to permanently hold the bones in the supine lithotomy position. The other organs and the 
tandem-ring applicator were attached to the bones and the base plate, so that they can be placed and removed frequently in a reproducible manner.

\section{The pelvic bone preparation}

A TecTake human skeleton anatomical model life size $181 \mathrm{~cm}$ plus poster plus bonnet was purchased from the TecTake prototyping company (UK). The pelvic bones, L4, L5 vertebral bodies with their cartilages and femoral heads were separated from the other parts of the skeleton in the mechanical workshop of the University of Surrey. After disassembling every part of the pelvic bone, a layer of ModRac plaster of Paris bandage roll with 2-3 mm thickness was adhered to them and then covered with a layer of plaster of Paris powder with approximately $1 \mathrm{~mm}$ thickness to make cortical bone equivalent material. Then, the bones were painted with oil-based gloss paint to waterproof the bones. Eventually, they were reassembled to the right shape of the pelvis again.

\section{D-printed internal organ construction and printing} procedure

Since the direct use of patient scan was so complicated for the practicality of in vivo dosimetry, we needed slightly simplified version of organs to create holes on their surface for place and wrap the threaded beads over them. Therefore, the internal organ shapes (bladder, rectum, sigmoid, and uterus) were initially made with a kind of modelling clay by using female pelvic anatomical references.

In the external radiotherapy energy range, Compton interactions play a key role in photon attenuation, and dependent on the atomic number and the electron density of the attenuator, the initial hypothesis was because of the similarity of effective atomic number of modelling clay $\left(Z_{\text {eff }}=7.6\right)[20]$ and water $\left(Z_{\text {eff }}=7.4\right)$, this material is suitable for dosimetric purposes in therapeutic photon energies (we wanted to construct a low-cost phantom and use it both in external RT and brachytherapy). However, the $\mathrm{CT}$ images revealed that the CT number of the polymer clays was similar to the bone, which means that it affected the photon attenuation coefficients, leading to dose calculation errors in megavoltage energies. Hence, we changed our methods and the CT images of the patient were used to produce 3D-printed organs. Furthermore, we used the initial organs (built with clay) for fixing into the phantom, which was really important. Finally, they were replaced with the 3D-printed organs for the real measurement.

In the following, axial CT images of an anonymous 40-year-old female patient (supine position) were obtained as per normal departmental protocols, using a RT simulator CT scanner (Toshiba Asteion, 4-slice CT scanner). The internal organs, including uterus, bladder, rectum, and sigmoid were delineated by an expert medical physicist using Pinnacle TPS. The uterus was an oval shape, with an approximate size of 6,4 , and $10 \mathrm{~cm}^{3}$ (length, width, and height, respectively), the bladder was a conical shape, with an approximate size of 10,6, and $5 \mathrm{~cm}^{3}$ (length, width, and height, respectively), and rec- tum and sigmoid were bent cylindrical shapes with approximate sizes of $2 \mathrm{~cm}$ and $14 \mathrm{~cm}$ (diameter and length, respectively). The DICOM images with delineated structures were transferred into 3D slicer v.4.1 [21,22]. Within this software, the segmentation editor was used to select individual organs and produce 3D mesh objects, which were exported to stereolithography (STL) files. The STL files were then processed in Autodesk ${ }^{\circledR}$ Fusion $360^{\mathrm{TM}}$ vs. 2.0 (2018, Autodesk, San Rafael, CA, USA), that were used as a reference to construct native solid bodies in the software. Holes of $2.5 \mathrm{~mm}$ depth and $2.2 \mathrm{~mm}$ in diameter for the positioning of the silica beads were created on the body's surface. The holes had $5 \mathrm{~mm}$ center point spacing and followed the contours of the model, and slots were created between holes for the thread connecting them to lie. The organ models (Figure 2A) including the holes were then exported to STL for 3D printing. The printing parameters are summarized in Table 1.

In this study, ABS filaments were used to print the organs due to its density $\left(1.05 \mathrm{~g} / \mathrm{cm}^{3}\right)$ and other properties' similarity to water $[23,24]$. A fused deposition modelling (FDM) 3D printer (CubePro, 3D Systems Inc., USA) was chosen to print the models due to its ability to print ABS layer-by-layer to create a 3D object. The printer has a heated bed and enclosure, which aids in ABS printing.

\section{Phantom assembly, irradiation plan, and evaluation methods}

After assembling the phantom (Figure 2B) and placing it into the water tank, a helical CT scan was taken (Toshiba Asteion 4-slice CT scanner, tube voltage $120 \mathrm{kVp}$; tube current $100 \mathrm{~mA}$; 512, 512 pixels; $1 \mathrm{~mm}$ slice interval and thickness; Figure 2C). The images were imported into the Pinnacle TPS. To evaluate the feasibility of using the 3D-printed phantom for dosimetry, the phantom properties were compared with solid water. The mass density and CT numbers of organs were sampled, using the region-of-interest software tool within the planning system and compared with Pinnacle's lookup table.

Then, silica beads were threaded and placed on the organs' surface in a square grid with $1 \mathrm{~cm}$ resolution, and a helical CT scan was taken from the phantom. The bead TLDs were exposed to the CT scan, as it was not possible to assemble them again after the CT scan. To address the issue of beads undergoing CT scan before BT irradiation in the anthropomorphic phantom, 30 beads (in 3 groups of 10 beads) were placed near each organ in the water tank. After performing the scan, these beads were removed and processed separately. The obtained doses were subsequently deducted from the doses measured in beads from the delivered BT plan.

The images were imported into the Sagiplan ${ }^{\circledR}$ TPS (Eckert \& Ziegler GmbH, Germany) and a treatment plan was created to deliver 7 Gy prescribed to ICRU38 defined A-points in the above-mentioned phantom (Figure 3). 162, 150, 94, and 52 silica beads were located on the uterus, bladder, rectum, and sigmoid, respectively. The phantom with bead TLDs was irradiated with a HDR ${ }^{60} \mathrm{Co}$ source (Eckert \& Ziegler GmbH, Germany) according to the planned dose distribution (Figure 2D). Due to the rapid 


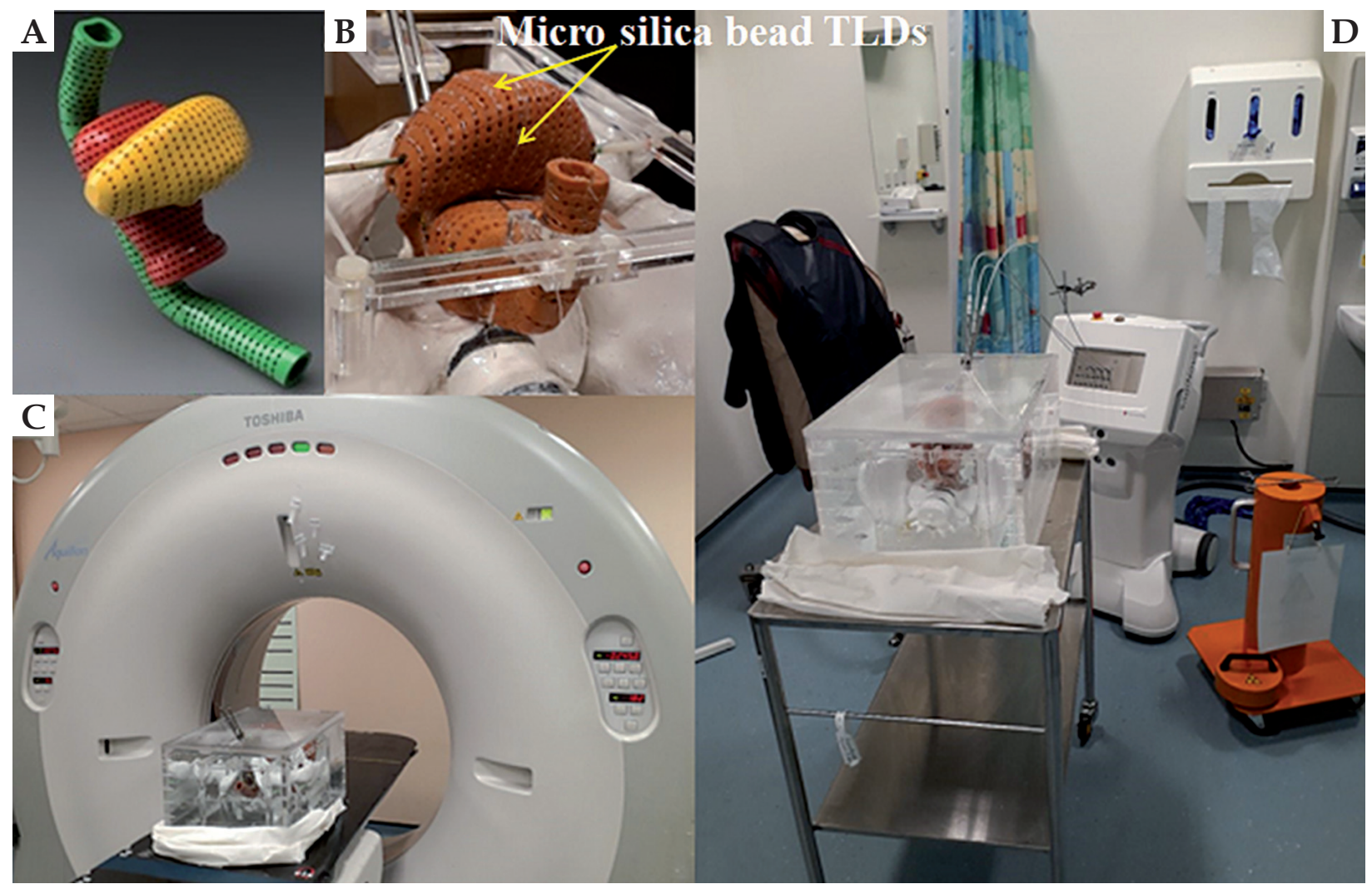

Fig. 2. A) 3D model view of the organs, B) 3D-printed organs with placement of thermoluminescent dosimeters (TLDs) at their surface, C) The anthropomorphic pelvis phantom in the water tank on the CT coach, D) The phantom in the water tank connected to high-dose-rate (HDR) unit

dose fall-off around BT sources, the beads were irradiated with different doses. According to the time, this represents a variation in dose rates. Additionally, in a publication by Jafari et al. it was shown that the response of beads is independent of the incident dose-rate [17]. The bead TLDs readout and the predicted dose extraction of corresponding points from TPS were performed. Finally, the percentage difference of mean doses and dose surface histograms (DSHs) of the measured doses were compared against the TPS calculated doses for the uterus as CTV, and for the bladder, rectum, and sigmoid as organs at risk (OARs).

The uncertainty budget of silica bead TLDs measurements at this phase, included $2.7 \%$ for determination of the element correction coefficients (ECCs) for each bead TLD, $0.1 \%$ for dose response linearity of silica bead TLD [15], $2.0 \%$ for TLD reading process [16], $0.5 \%$ for bead calibration [16], and $1 \%$ for energy dependency of silica beads [25], which leads to $3.5 \%(k=1)$ of combined standard uncertainty.

\section{Results}

\section{Evaluating photon beam perturbation}

Comparing organs doses according to DVHs

After calculating the dose distribution with the box 3D conformal plan, the dose volume histograms (DVHs) of the organs were extracted from the TPS, and the per-
Table 1. 3D-printing parameters to construct the pelvis phantom organs

\begin{tabular}{lc}
\hline Setting & Value \\
\hline Bed temperature & $40^{\circ} \mathrm{C}$ \\
\hline Extruder temperature & $160^{\circ} \mathrm{C}$ \\
\hline Layer height & $0.2 \mathrm{~mm}$ \\
\hline First layer height & $0.2 \mathrm{~mm}$ \\
\hline Vertical shells & $\mathrm{N} / \mathrm{A}$ \\
\hline Horizontal shells & $\mathrm{N} / \mathrm{A}$ \\
\hline Speed of print movement (perimeter) & $80.0 \mathrm{~mm} / \mathrm{s}$ \\
\hline Speed of print movement (external perimeter) & $50 \%$ \\
\hline Speed of print movement (infill) & $80 \%$ \\
\hline First layer print movement speed & $80.0 \mathrm{~mm} / \mathrm{s}$ \\
\hline Fill density & $90 \%$ \\
\hline Fill pattern & Solid \\
\hline Fill angle & 0 \\
\hline Nozzle diameter & $0.40 \mathrm{~mm}$ \\
\hline Filament diameter & $1.5 \mathrm{~mm}$ \\
\hline
\end{tabular}




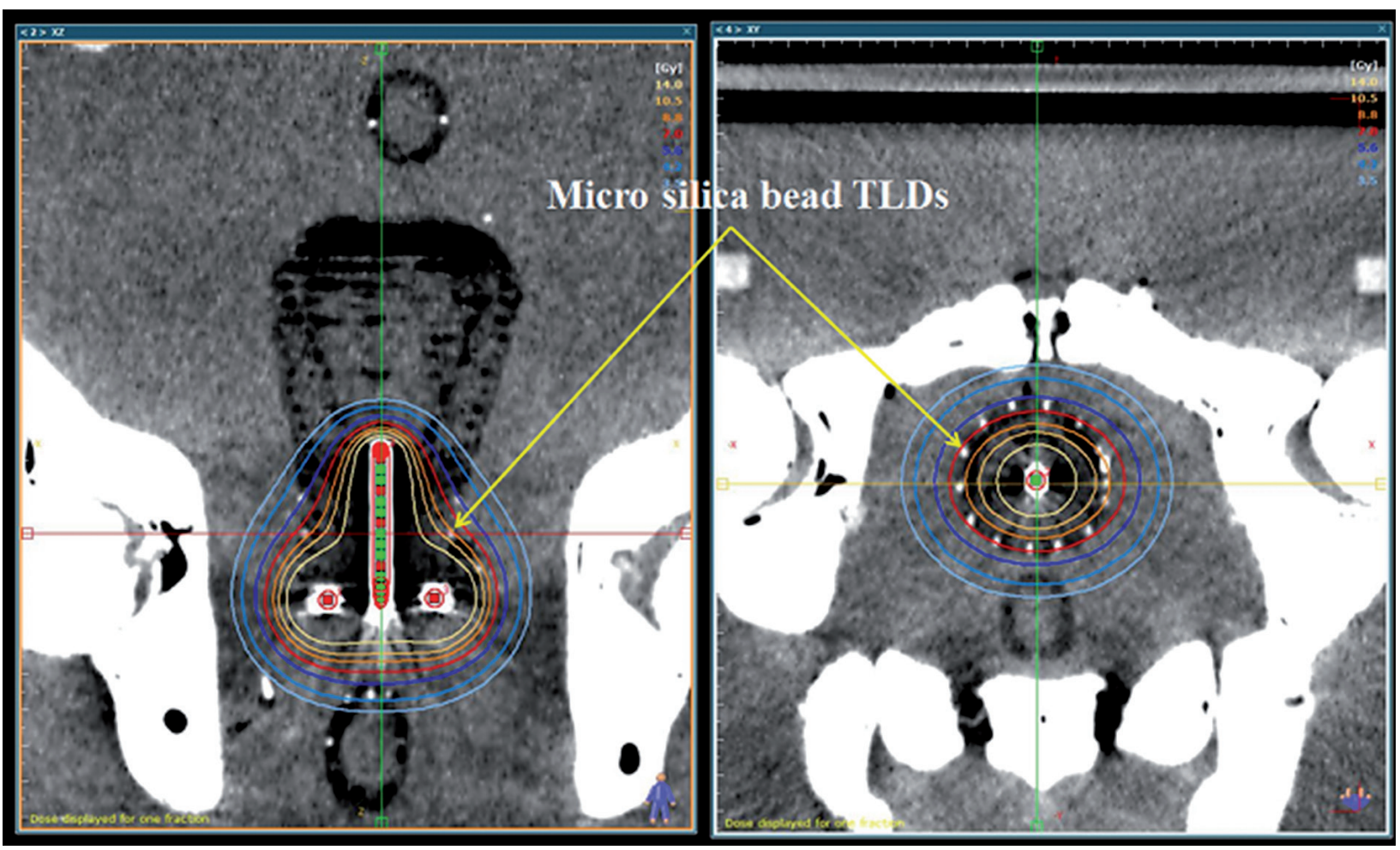

Fig. 3. High-dose-rate (HDR) plan on the CT image of the phantom (coronal and axial view); each thermoluminescent dosimeter (TLD) is clearly visible

Table 2. Result of the organs' dose obtained at different silica bead resolution on the syringe according to DVH (the doses are in cGy)

\begin{tabular}{lcccc} 
Organs (mean dose) & No bead & Hres $^{\mathrm{a}}(2.5 \mathrm{~mm})$ & Mres $^{\mathrm{b}}(5 \mathrm{~mm})$ & Lres $^{\mathrm{c}}\left(7.5 \mathrm{~mm}^{\prime}\right)$ \\
\hline Rectum & 3449.59 & 3445.89 & 3448.44 & 3449.36 \\
\hline Rectal wall & 3639.23 & 3634.43 & 3638.47 & 3638.79 \\
\hline Bladder & 3578.50 & 3576.96 & 3578.53 & 3578.50 \\
\hline CTV & 4522.45 & 4519.65 & 4522.21 & 4522.44 \\
\hline PTV & 4491.17 & 4488.50 & 4490.79 & 4491.14
\end{tabular}

ahigh resolution, bmedium resolution, clow resolution

centage differences of the delivered dose to organs with the presence of silica beads (with different resolutions) and without beads were obtained (Tables 2 and 3). According to these numbers, there was no significant difference between the absorbed doses of organs with silica beads in various SR.

\section{Gamma evaluation method}

Low et al. [26] defined the gamma index $(\gamma)$ to compare the distributions of calculated and measured doses. In this part of the project, $\gamma$ index evaluation was used to find out whether the silica beads have any effect on radiation transmission and radiation field perturbation. VeriSoft software (v.7.1, PTW, Germany) was used to com-
Table 3. Result of percentage dose differences of the organs at different silica bead resolution on the syringe according to DVH

\begin{tabular}{lccc} 
& $\begin{array}{c}\text { (Hres } \\
\text { no bead) }\end{array}$ & $\begin{array}{c}\text { (Mres }^{\mathrm{b}} \text { vs. } \\
\text { no bead) }\end{array}$ & $\begin{array}{c}\text { (Lres }{ }^{\mathrm{c}} \text { vs. } \\
\text { no bead })\end{array}$ \\
\hline Rectum & 0.11 & 0.03 & 0.00 \\
\hline Rectal wall & 0.13 & 0.02 & 0.01 \\
\hline Bladder & 0.04 & 0.00 & 0.00 \\
\hline CTV & 0.06 & 0.01 & 0.00 \\
\hline PTV & 0.06 & 0.01 & 0.00
\end{tabular}

ahigh resolution, ${ }^{b}$ medium resolution, clow resolution 
Table 4. Result of the comparing planes with and without beads at different source to plane distance (SPDs) and different bead resolutions on the syringe with Verisoft software $(3 \%, 3 \mathrm{~mm}$ criteria)

\begin{tabular}{lll} 
& $\begin{array}{c}\gamma \text { index } \\
\text { (mean) }\end{array}$ & $\begin{array}{c}\text { \% difference } \\
\text { (mean) }\end{array}$ \\
\hline $\mathrm{SPD}=102 \mathrm{~cm}$ & 0.071 & 0.246 \\
\hline High resolution $(2.5 \mathrm{~mm})$ & 0.008 & 0.026 \\
\hline Medium resolution $(5.0 \mathrm{~mm})$ & 0.001 & 0.004 \\
\hline Low resolution $(7.5 \mathrm{~mm})$ & & \\
\hline $\mathrm{SPD}=100 \mathrm{~cm}$ & 0.080 & 0.299 \\
\hline High resolution $(2.5 \mathrm{~mm})$ & 0.014 & 0.053 \\
\hline Medium resolution $(5.0 \mathrm{~mm})$ & 0.009 \\
\hline Low resolution $(7.5 \mathrm{~mm})$ & 0.003 & \\
\hline $\mathrm{SPD}=99 \mathrm{~cm}$ & & 0.952 \\
\hline High resolution $(2.5 \mathrm{~mm})$ & 0.064 & 0.676 \\
\hline Medium resolution $(5.0 \mathrm{~mm})$ & 0.007 & 0.659 \\
\hline Low resolution $(7.5 \mathrm{~mm})$ & 0.003 &
\end{tabular}

pare the dose of the plane with no beads as a reference, and the planes including beads with different SR at different distances from the source (different SPD). Tables 4 and 5 present the results of these comparisons.

According to these tables, the 2D- $\gamma$ analysis on the syringe showed more than $99 \%$ points passed $3 \%$ and $3 \mathrm{~mm}$ (also $2 \%$ and $2 \mathrm{~mm}$ ) criteria at all SPDs, which means that external treatment planning with $0.1 \mathrm{~mm}$ dose grid resolution did not present any significant perturbation effects due to the presence of bead TLDs with different SR.

\section{Evaluation and measurement on the phantom}

\section{Physical evaluation}

We found that the 3D-printed internal organ materials have an average physical density $\rho$, electron density (relative to water), and CT numbers of $0.95 \pm 0.01 \mathrm{~g} / \mathrm{cm}^{3}$, $0.97 \pm 0.01$, and $959 \pm 5$, respectively (solid water: CT number $=1012,0.944<\rho<1.044 \mathrm{~g} / \mathrm{cm}^{3}$ ), based on a Pinnacle's lookup table.

\section{Dosimetric evaluation}

Figure 4 shows the DSHs of measured doses with TLDs in comparison with those of the TPS calculation. By calculating the integral dose for the organs, the percentage differences were found to be $-1.5 \%, 2 \%, 5 \%$, and $10 \%$ for uterus, rectum, bladder, and sigmoid, respectively. Also, the results showed that the mean absolute dose difference of TLD measurements compared to the TPS calculations were $-4.5 \pm 19.5 \mathrm{cGy}, 4.8 \pm 9.5 \mathrm{cGy}, 4.3 \pm 8.7 \mathrm{cGy}$, and $2.9 \pm 5.1 \mathrm{cGy}(1 \mathrm{SD})$ in the uterus, rectum, bladder, and
Table 5. Result of the comparing planes with and without beads at different source to plane distance (SPDs) and different bead resolutions on the syringe with Verisoft software $(2 \%, 2 \mathrm{~mm}$ criteria)

\begin{tabular}{lll} 
& $\begin{array}{c}\gamma \text { index } \\
\text { (mean) }\end{array}$ & $\begin{array}{c}\text { \% difference } \\
\text { (mean) }\end{array}$ \\
\hline $\mathrm{SPD}=102 \mathrm{~cm}$ & 0.107 & 0.246 \\
\hline High resolution $(2.5 \mathrm{~mm})$ & 0.011 & 0.026 \\
\hline Medium resolution $(5.0 \mathrm{~mm})$ & 0.002 & 0.004 \\
\hline Low resolution $(7.5 \mathrm{~mm})$ & & \\
\hline $\mathrm{SPD}=100 \mathrm{~cm}$ & 0.120 & 0.299 \\
\hline High resolution $(2.5 \mathrm{~mm})$ & 0.021 & 0.053 \\
\hline Medium resolution $(5.0 \mathrm{~mm})$ & 0.009 \\
\hline Low resolution $(7.5 \mathrm{~mm})$ & 0.004 & \\
\hline SPD $=99 \mathrm{~cm}$ & & 0.952 \\
\hline High resolution $(2.5 \mathrm{~mm})$ & 0.096 & 0.676 \\
\hline Medium resolution $(5.0 \mathrm{~mm})$ & 0.010 & 0.659 \\
\hline Low resolution $(7.5 \mathrm{~mm})$ & 0.004 &
\end{tabular}

sigmoid, respectively, which were $-1.4 \pm 6.1 \%, 2.1 \pm 4.1 \%$, $5.2 \pm 10.6 \%$, and $10.9 \pm 18.7 \%$ ( 1 SD) of the TPS mean doses (negative sign indicates larger measured dose). The mean doses of TLD reading were higher than those calculated by the TPS in the uterus. Although the percentage differences were higher for the beads located on the bladder and sigmoid, this occurred for regions with mean doses < 1 Gy (at distances approximately $10 \mathrm{~cm}$ and more from the source), which is not important in real BT clinical situation. Finding high variation in percentage dose differences was due to the steep dose fall-off, which occurs in BT radiation fields. In this study, this was more evident in a low-dose region, since a small difference in absolute dose led to a high percentage of difference.

On the other hand, these high differences may arise from the energy dependency of bead TLDs' response to low energy photons. According to Jafari et al. [18], the greater energy dependency of bead TLDs in a lower energy range $(<100 \mathrm{keV})$ can be explained by the non-soft tissue equivalence (silicon-based) of beads, as the photoelectric process is the dominant interaction in this region. Therefore, the attenuation coefficient of bead TLDs and their energy response at far distances from the BT source may need more investigation using Monte Carlo simulation.

\section{Timeline and costs}

The timeline and cost of the organs are shown in Table 6 . The cost for the human body skeleton was 100 GBP, the water tank and base plate came to 105 GBP, and 60 GBP for the ABS cartridge. Therefore, the total price 

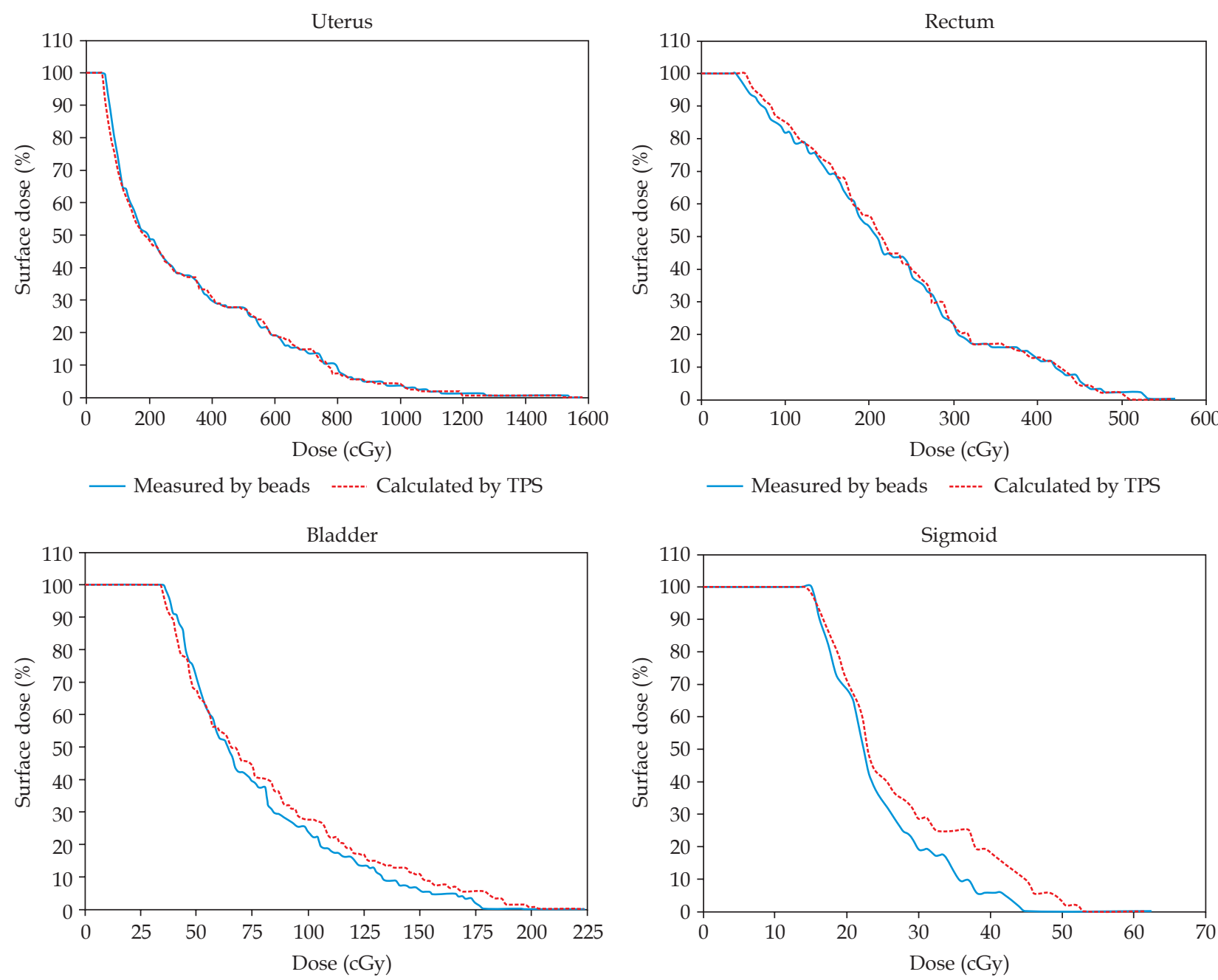

— Measured by beads -.--.- Calculated by TPS

— Measured by beads ...--. Calculated by TPS

Fig. 4. Dose surface histograms obtained by thermoluminescent dosimeters (TLDs) readout and point doses calculation of treatment planning system (TPS)

was 385 GBP for materials, which was comparable to other commercial phantoms.

The weight of the water tank and base plate with bones and organs were approximately $5 \mathrm{~kg}$. The weights of them with full water tank were approximately $40 \mathrm{~kg}$.

\section{Discussion}

In this study, a phantom was needed to place the silica beads TLDs similar to real organ position, but it was found that commercial phantoms were not suitable for this purpose; so, it was decided to construct an anthropomorphic and low-cost pelvis phantom for the use in $3 \mathrm{D}$ dosimetry of external RT and BT.

Phantoms used in radiation dosimetry should be made of materials that render the same radiological properties as the real organ tissues, especially in terms of the radiation attenuation. Although various $3 \mathrm{D}$-printing materials have been studied $[2,8,9,13,23,24]$, there is no agreement on printing parameters and settings used, such as the in-
Table 6. Print statistics for each individual internal organ in the anthropomorphic pelvis phantom

\begin{tabular}{lccc} 
Organ & $\begin{array}{c}\text { Print } \\
\text { time }(\mathrm{h})\end{array}$ & Mass (g) & $\begin{array}{c}\text { Print cost } \\
\text { (GBP) }\end{array}$ \\
\hline Bladder & 5.56 & 42.3 & 40 \\
\hline Uterus & 5.42 & 85.2 & 40 \\
\hline Rectum and sigmoid & 7.07 & 27.1 & 40 \\
\hline Total & 18.05 & 154.6 & 120
\end{tabular}

fill density and infill pattern of materials with different 3D-printing systems. It appears that depending on the intended application, specific settings should be considered to use with any 3D printer and material. Therefore, ABS and polylactic acid (PLA) plastics were tested with different fill densities. Eventually, it was found that ABS with 
$90 \%$ fill density was best suited for mimicking human soft tissues by using the CubePro 3D printer. Bladder and rectum were 3D printed as hollow objects, with a $90 \%$ infilled outer boundary (with thickness about 5-6 $\mathrm{mm}$ ) and the uterus was solid.

The constructed phantom and organs, which are shown in Figure 2B, were simple to assemble; organs could be repositioned with no discernible displacement. The values of CT number and the other parameters of the organs indicate agreement between them, with relevant tissues within the tolerances.

In this project, it was necessary to obtain clinically relevant SR required for placing the silica beads at the organs' surface, which does not cause significant perturbation effect. Locating the dosimeter on the path of central axis of the radiation field is a popular method for measuring beam perturbation. Until now, the beam perturbation effect was measured for different dosimeters specially diodes $[27,28]$, which are the commonly used one for in vivo dosimetry. Since it was desired to evaluate this effect in the presence of a large number of bead TLDs, a special test was designed. The bead TLDs were arranged on the surface of a syringe with different SR and placed in the water tank for CT scanning. As the results showed, despite the beads TLD's relatively high density and atomic number, they minimally perturb the radiation field due to their small size, causing no considerable decrease or increase in dose.

Silva et al. in 2015 performed a dosimetric study on an anthropomorphic phantom to investigate a bladder dose. They exposed radiochromic film (strips with a width of $1 \mathrm{~cm}$ ) and optically simulated luminescent dosimeters (OSLDs) with a diameter of $5.0 \mathrm{~mm}$ and thickness of $1.0 \mathrm{~mm}$, using a HDR ${ }^{192} \mathrm{Ir}$ source on the surface of two 3D-printed bladders. The study has shown that approximately $83 \%$ and $66.7 \%$ of the doses measured using film exhibited discrepancies lower than $10 \%$ and $5 \%$ with the results obtained by TPS. They have estimated the expanded uncertainty of $8.6 \%$ in the film calibration procedure. Also, approximately $92 \%$ of OSLD results (36 measurement points) illustrated percentage differences lower than $10 \%$ [13]. In this study, although most of the results obtained using films and OSL dosimeters were in agreement with those acquired by the TPS, significant differences occurred in some points, such as between the film and TPS $(25.3 \%)$ and the film and OSLD $(-22.1 \%)$ on the anterior wall of bladder.

In another GYN patient in vivo dosimetry performed by Sharma et al. [29], OSLDs (nanodots with a disk sensible volume of $5 \mathrm{~mm}$ in diameter and $0.2 \mathrm{~mm}$ thick) were placed on modified rectal retractors and exposed with a HDR ${ }^{192}$ Ir source. They found that the range of differences between the measured and TPS calculated doses (20 points) was $-10.1 \%$ to $13.7 \%$ with Acuros algorithm and $-14.9 \%$ to $11.6 \%$ with TG-43 algorithm.

According to the number of the bead TLDs, which was utilized for high resolution surface dosimetry of the pelvis organs in the unique constructed phantom, the results may not be comparable with the other point dosimeters, but they can render a relative viewpoint to the leadership.
Eventually, summarizing dose distribution throughout the organ volumes is customarily estimated by using DVHs. For hollow organs, like the rectum and bladder, the dose received by these organs' contents is not important, therefore some researchers $[30,31,32,33]$ suggested working with dose wall histograms (DWHs) and DSHs, which are more clinically relevant to evaluate the dose distribution created over these organs. In this regard, by using a large number of bead TLDs on the organs' surfaces and performing 3D dosimetry, obtaining the DSHs of the organs has been achieved.

\section{Conclusions}

In this study, an anthropomorphic pelvis phantom was successfully constructed for performing 3D dosimetry using silica bead TLDs. The phantom consists of pelvic organs, which are relevant in GYN treatment, and can be used for external RT and BT. A 90\% ABS infill density was found to result in a material suitable for modelling soft tissue, with CT scan images showing good contrast between anatomical structures. The constraint of the current project was handling and reading out a large number of silica beads, which were labor-intensive and time-consuming procedures. This inhibited performing BT dosimetry under different doses and treatment plans, which is a limitation of this study. More investigation might be helpful to provide better understanding of the behavior of the phantom under different clinical conditions. Although the bead TLD responses were assessed already in external RT by Jafari et al. $[15,16,17,34]$, it could be better to execute an external RT plan on this phantom within the presence of the silica beads.

The results of the current project demonstrate that the developed phantom and also commercially available silica bead TLDs are suitable for dosimetric studies of GYN treatments. Also, for the first time, plotting DSHs using the measurements of a large number of dosimeters is possible.

\section{Acknowledgments}

The first and the last authors would like to express their deepest gratitude and thanks to the University of Surrey for the IAS (Institute of Advanced Studies) Research Scholarship that made this research project possible. The assistance of TrueInvivo Ltd. Company is acknowledged for providing and facilitating the use of micro-silica bead TLDs. The authors would also like to convey thanks to the radiotherapy department of the Portsmouth Hospitals NHS Trust for providing the facilities for irradiations.

\section{Disclosure}

Jafari S. is founder and CTO of TrueInvivo Ltd., UK. The rest of authors report no conflict of interest.

\section{References}

1. Nattagh K, Siauw T, Pouliot J et al. A training phantom for ultrasound-guided needle insertion and suturing. Brachytherapy 2014; 13: 413-419. 
2. Leary M, Kron T, Keller C et al. Additive manufacture of custom radiation dosimetry phantoms: An automated method compatible with commercial polymer 3D printers. Materials Design 2015; 86: 487-499.

3. DeWerd LA, Kissick M (Eds.). The phantoms of medical and health physics. Springer, New York 2014.

4. Ebert M, Harrison KM, Howlett SJ et al. Dosimetric intercomparison for multicenter clinical trials using a patient-based anatomic pelvic phantom. Med Phys 2011; 38: 5167-5175.

5. Yea JW, Park JW, Kim SK et al. Feasibility of a 3D-printed anthropomorphic patient-specific head phantom for patientspecific quality assurance of intensity-modulated radiotherapy. PLoS One 2017; 12: e0181560.

6. Kron T, Hamilton C, Roff M et al. Dosimetric intercomparison for two Australasian clinical trials using an anthropomorphic phantom. Int J Radiat Oncol Biol Phys 2002; 52: 566-579.

7. Opp D, Nelms BE, Zhang G et al. Validation of measurement-guided 3D VMAT dose reconstruction on a heterogeneous anthropomorphic phantom. J Appl Clin Med Phys 2013; 14: 70-84.

8. Kamomae T, Shimizu H, Nakaya $\mathrm{T}$ et al. Three-dimensional printer-generated patient-specific phantom for artificial in vivo dosimetry in radiotherapy quality assurance. Phys Med 2017; 44: 205-211.

9. Craft DF, Howell RM. Preparation and fabrication of a fullscale, sagittal-sliced, 3D-printed, patient-specific radiotherapy phantom. J Appl Clin Med Phys 2017; 18: 285-292.

10. Ehler ED, Barney BM, Higgins PD et al. Patient specific 3D printed phantom for IMRT quality assurance. Phys Med Biol 2014; 59: 5763-5773.

11. Nazarnejad M, Mahdavi SR, Sadeghi M et al. Developing a verification and training phantom for gynecological brachytherapy system. Iran J Med Phys 2012; 9: 33-40.

12. de Almeida CE, Rodriguez M, Vianello E et al. An anthropomorphic phantom for quality assurance and training in gynaecological brachytherapy. Radiother Oncol 2002; 63: 75-81.

13. Silva R, Belinato W, Macedo LE et al. Anthropomorphic phantom to investigate the bladder dose in gynecological highdose-rate brachytherapy. Brachytherapy 2015; 14: 633-641.

14. Guiral P, Ribouton J, Jalade P et al. Design and testing of a phantom and instrumented gynecological applicator based on $\mathrm{GaN}$ dosimeter for use in high dose rate brachytherapy quality assurance. Med Phys 2016; 43: 5240-5251.

15. Jafari SM, Jordan TJ, Distefano G et al. Feasibility of using glass-bead thermoluminescent dosimeters for radiotherapy treatment plan verification. Br J Radiol 2015; 88: 20140804

16. Palmer AL, Jafari SM, Mone I et al. Evaluation and clinical implementation of in vivo dosimetry for $\mathrm{kV}$ radiotherapy using radiochromic film and micro-silica bead thermoluminescent detectors. Phys Med 2017; 42: 47-54.

17. Jafari SM, Bradley DA, Gouldstone CA et al. Low-cost commercial glass beads as dosimeters in radiotherapy. Radiat Phys Chem 2014; 97: 95-101.

18. Jafari S, Jordan TJ, Hussein M et al. Energy response of glass bead TLDs irradiated with radiation therapy beams. Radiat Phys Chem 2014; 104: 208-211.

19. Khan FM, Gibbons JP. Khan's the physics of radiation therapy. Lippincott Williams \& Wilkins, Philadelphia 2014.

20. Singh V, Badiger N, Kucuk N. Assessment of methods for estimation of effective atomic numbers of common human organ and tissue substitutes: waxes, plastics and polymers. Radioprotection 2014; 49: 115-121.

21. Kikinis R, Pieper SD, Vosburgh KG. 3D Slicer: a platform for subject-specific image analysis, visualization, and clinical support. In: Intraoperative imaging and image-guided therapy. Springer 2014; 277-289.
22. Fedorov A, Beichel R, Kalpathy-Cramer J et al. 3D Slicer as an image computing platform for the Quantitative Imaging Network. Magn Reson Imaging 2012; 30: 1323-1341.

23. Alssabbagh M, Tajuddin AA, Manap MA et al. Evaluation of nine $3 \mathrm{D}$ printing materials as tissue equivalent materials in terms of mass attenuation coefficient and mass density. Int J Adv Appl Sci 2017; 4: 168-173.

24. Kairn T, Crowe S, Markwell T. Use of 3D printed materials as tissue-equivalent phantoms. In World Congress on Medical Physics and Biomedical Engineering, June 7-12, 2015, Toronto, Canada. Springer, 2015.

25. Douralis A. Verification of high dose rate brachytherapy dosimetry and treatment delivery using micro silica bead thermoluminescent detectors. Master of Science dissertation, University of Surrey, 2017.

26. Low DA, Harms WB, Mutic S et al. A technique for the quantitative evaluation of dose distributions. Med Phys 1998; 25: 656-661.

27. Alecu R, Feldmeier JJ, Alecu M. Dose perturbations due to in vivo dosimetry with diodes. Radiother Oncol 1997; 42: 289-291.

28. Sen A, Parsai EI, McNeeley SW et al. Quantitative assessment of beam perturbations caused by silicon diodes used for in vivo dosimetry. Int J Radiat Oncol Biol Phys 1996; 36: 205-211.

29. Sharma R, Jursinic PA. In vivo measurements for high dose rate brachytherapy with optically stimulated luminescent dosimeters. Med Phys 2013; 40: 071730.

30. Fenwick JD, Khoo VS, Nahum AE et al. Correlations between dose-surface histograms and the incidence of long-term rectal bleeding following conformal or conventional radiotherapy treatment of prostate cancer. Int J Radiat Oncol Biol Phys 2001; 49: 473-480.

31. Maggio A, Carillo V, Cozzarini C et al. Impact of the radiotherapy technique on the correlation between dose-volume histograms of the bladder wall defined on MRI imaging and dose-volume/surface histograms in prostate cancer patients. Phys Med Biol 2013; 58: N115-123.

32. Carillo V, Cozzarini C, Rancati T et al. Relationships between bladder dose-volume/surface histograms and acute urinary toxicity after radiotherapy for prostate cancer. Radiother Oncol 2014; 111: 100-105.

33. Yanagi T, Kamada T, Tsuji H et al. Dose-volume histogram and dose-surface histogram analysis for skin reactions to carbon ion radiotherapy for bone and soft tissue sarcoma. Radiother Oncol 2010; 95: 60-65.

34. Jafari S, Distefano G, Lee J et al. Feasibility study of silica bead thermoluminescence detectors (TLDs) in an external radiotherapy dosimetry audit programme. Radiat Phys Chem 2017; 141: 251-256 